\title{
Real-World Extensions to a Production Scheduling Algorithm Based on Lagrangian Relaxation
}

\author{
Y. Narahari \\ Computer Science and Automation \\ Indian Institute of Science \\ Bangalore 560012 - INDIA
}

\author{
Sundar Ram Vedula \\ Mechanical Engineering \\ Indian Institute of Technology \\ Madras 600036 - INDIA
}

\begin{abstract}
In this article, we develop three efficient extensions to a recent production scheduling algorithm based on Lagrangian relaxation [9]. These extensions handle the following real-world features:

- stochastic availability of raw material,

- reworking and scrapping of parts, and

- incorporating set-up costs and set-up times in the scheduling of multiclass manufacturing systems

The first two extensions yield on-line algorithms which are illustrated using a 89-job scheduling problem. The third extension uses an algorithmic procedure to insert switchovers to obtain a good schedule and is illustrated for a two-class, 89-job scheduling problem.
\end{abstract}

\section{Introduction}

Discrete activity scheduling $[2,3,7,12]$ is probably the most important issue in the design and operation of manufacturing systems. This paper is concerned with deterministic machine scheduling in a system comprising parallel identical machines. We present three realworld extensions to a recent production scheduling algorithm based on Lagrangian relaxation [9]. The extensions handle the following real-world features:

1. Stochastic availability of raw material

2. Reworking and scrapping of parts

3. Switchover costs and set-up times encountered in the scheduling of multiclass manufacturing systems.

These extensions are illustrated for a detailed 89 -job example adapted from a real-world problem presented in [9].

\subsection{Schedule Generation Using La- grangian Relaxation}

The theory of sequencing and scheduling, more than any other area in Operations Research, is characterized by a virtually unlimited number of problem types $[2,7]$. Recently, in the area of deterministic machine scheduling, scheduling algorithms based on Lagrangian relaxation $[1,8]$ have been proposed and have proved to be highly effective in real-world factory situations. These Lagrangian relaxation-based algorithms have been reviewed in [6]. Luh, Hoitomt, Max, and Pattipati [9] presented the first of these algorithms for the case of scheduling single operation jobs on parallel identical machines. Later, the approach was extended to the case of multiple-operation jobs with precedence constraints [5]. More recently, the approach has been extended to general job shop scheduling problems [4, 6]. The algorithms use a decomposable objective function such as the total weighted tardiness and obtain the solution by solving independent, job-level subproblems.

We now give a brief description of the basic technique that we employ in this paper. This technique is due to Luh, Hoitomt, Max, and Pattipati [9]. The method is used for the nonpreemptive discrete time scheduling of independent jobs on identical, parallel machines and is based on the Lagrangian relaxation algorithm $[1,8]$. The input variables are:

$N$ : Number of jobs

$K$ : Time horizon for scheduling

$w_{1}, \cdots w_{N}$ : Weights (relative importance) of jobs

$t_{1}, \cdots, t_{N}$ : Processing times of the jobs

$D_{1}, \cdots, D_{N}:$ Due dates of the jobs

$M_{1}, \cdots, M_{K}$ : Numbers of machines available at discrete time instants $1,2, \cdots, K$, respectively.

The decision variables are $B_{1}, \cdots, B_{N}$, the beginning times for the jobs. Now, define, for $i=1, \cdots, N$, and $k=1, \cdots, K$,

$\delta_{i k}=1$ if job $i$ is undergoing processing at time $k$ 
$\delta_{i k}=0$ if job $i$ is not undergoing processing at time $k$

$C_{i}=$ Completion time of job $i$

$T_{i}=\max \left\{0, C_{i}-D_{i}\right\}$

$T_{i}$ represents the tardiness of job $i$. The objective function of interest is

$$
J=\sum_{i=1}^{N} w_{i} T_{i}
$$

which is the weighted tardiness criterion. This criterion takes into account the relative importance of jobs, and the importance of meeting due dates. The scheduling problem can now be stated as follows.

$$
\min _{\left\{B_{i}\right\}} J \text { with } J=\sum_{i=1}^{N} w_{i} T_{i}
$$

subject to capacity constraints:

$$
\sum_{i=1}^{N} \delta_{i k} \leq M_{k}, k=1, \cdots, K
$$

and processing time requirements:

$$
C_{i}-B_{i}+1=t_{i}, i=1, \cdots, N
$$

The problem is solved by maximizing the dual function which is obtained by using a set of multipliers to relax the capacity constraints. The dual problem can be decomposed into $N$ subproblems, one for each job. The computational complexity of each subproblem is linear in $K$. Using these $N$ solutions, the dual problem is solved using a subgradient method [10]. The stopping criterion used in the above subgradient method does not always yield a feasible schedule, in the sense that the capacity constraints might be violated for a few time slots [9]. To construct a feasible schedule, a greedy heuristic based on the list scheduling concept is used.

\subsection{Extensions Proposed}

In this paper, we consider the LR (Lagrangian relaxation) approach presented in [9] and develop extensions to address three real-world features in scheduling. These features are different from the extensions to the algorithm presented in [9].

The first extension proposed handles delayed or stochastic availability of raw material. Since raw materials are usually procured from sources external to the machine shop, one is never sure unless the raw material is in hand. The LR algorithm of Luh et al [9] handles in a limited way the feature of delayed availability of raw material, by specifying earliest start times, which are however assumed to be known in advance. In a stochastic environment where the raw material keeps arriving into the shop at time instants not known in advance, the earliest start times cannot capture fully the situation.

The second extension seeks to address the rescheduling problem that arises when processed parts are either rejected or sent for reworking after periodic inspections. Parts identified for reworking cause extra load on the system whereas every rejected part entails complete reprocessing and also material waste. As a result, the originally drawn schedule may not be the best any more and rescheduling becomes necessary.

The problem of set-ups in multiclass manufacturing system is addressed by the third extension we propose. Our method enables set-up costs to be included in the scheduling procedure. For this, we first obtain a schedule without set-up operations (using the LR-algorithm [9]) and modify this schedule using a simple heuristic algorithm, to include the effect of set-up times and set-up costs.

The paper is organized as follows. In Section 2, we present the input data for a 89 -job scheduling problem, which is used for illustrating the three extensions proposed. This data is adapted from a real-world situation described in [9]. The three extensions and relevant numerical experimentation are presented in Sections 3,4 , and 5 .

\section{Data for Scheduling Example}

Table 1 shows the input data for a 89 -job system. This data is the same as in [9] and is a real-world data taken from the tool and die workcenter of Pratt and Whitney's Development operations shop. We assume that the machine shop has 4 identical machines (the data in [9] has 42 machines). The first column shows the job number; the second column, the job weight; the third column, the job processing time; and the fourth column, the job due date. The fifth column is a job class column introduced in this paper, specially for discussing the set-up problem in Section 5 . In the three sections that follow, we apply the proposed extensions to the above data by introducing specific changes needed. 


\begin{tabular}{|c|c|c|c|c|c|c|c|c|c|}
\hline$i$ & $w_{i}$ & $t_{i}$ & $D_{i}$ & $\mathrm{cl}$ & $i$ & $w_{i}$ & $t_{i}$ & $D_{i}$ & $\mathrm{cl}$ \\
\hline 1 & 1 & 1 & 0 & $\mathrm{~A}$ & 2 & 1 & 2 & 15 & $\mathrm{~A}$ \\
\hline 3 & 9 & 1 & 12 & $\mathbf{A}$ & 4 & 1 & 1 & 13 & A \\
\hline 5 & 1 & 1 & 1 & $\mathbf{A}$ & 6 & 1 & 1 & -3 & A \\
\hline 7 & 1 & 2 & -2 & A & 8 & 1 & 4 & 0 & B \\
\hline 9 & 1 & 3 & 9 & B & 10 & 1 & 1 & 7 & A \\
\hline 11 & 1 & 1 & 8 & A & 12 & 1 & 2 & 13 & A \\
\hline 13 & 6 & 5 & 19 & B & 14 & 1 & 1 & 2 & A \\
\hline 15 & 1 & 1 & 7 & A & 16 & 1 & 2 & -1 & A \\
\hline 17 & 5 & 1 & 1 & A & 18 & 1 & 3 & 0 & B \\
\hline 19 & 1 & 1 & 5 & A & 20 & 9 & 10 & 9 & B \\
\hline 21 & 1 & 1 & 1 & A & 22 & 1 & 2 & 8 & A \\
\hline 23 & 1 & 3 & 0 & B & 24 & 1 & 7 & 3 & B \\
\hline 25 & 6 & 8 & 60 & B & 26 & 1 & 9 & 8 & B \\
\hline 27 & 1 & 2 & -1 & A & 28 & 1 & 3 & 3 & B \\
\hline 29 & 1 & 2 & 0 & $\mathbf{A}$ & 30 & 6 & 1 & 2 & A \\
\hline 31 & 9 & 1 & -1 & A & 32 & 1 & 1 & 7 & A \\
\hline 33 & 1 & 11 & 11 & B & 34 & 1 & 2 & 15 & A \\
\hline 35 & 1 & 16 & 24 & B & 36 & 16 & 5 & 2 & B \\
\hline 37 & 1 & 3 & 25 & B & 38 & 1 & 3 & 24 & B \\
\hline 39 & 1 & 3 & 20 & B & 40 & 1 & 4 & 18 & B \\
\hline 41 & 1 & 1 & 2 & $\mathbf{A}$ & 42 & 1 & 3 & 2 & B \\
\hline 43 & 6 & 5 & 10 & B & 44 & 1 & 3 & 7 & B \\
\hline 45 & 1 & 1 & 7 & A & 46 & 1 & 3 & 2 & B \\
\hline 47 & 1 & 2 & 7 & $\mathbf{A}$ & 48 & 1 & 1 & 7 & A \\
\hline 49 & 1 & 1 & 8 & A & 50 & 1 & 2 & 7 & A \\
\hline 51 & 1 & 2 & 7 & $\mathbf{A}$ & 52 & 1 & 1 & 10 & A \\
\hline 53 & 1 & 1 & 9 & A & 54 & 1 & 3 & 2 & B \\
\hline 55 & 9 & 4 & 1 & B & 56 & 1 & 3 & 9 & B \\
\hline 57 & 1 & 3 & 2 & B & 58 & 1 & 2 & -1 & A \\
\hline 59 & 1 & 1 & 10 & A & 60 & 1 & 20 & 24 & $\mathrm{~B}$ \\
\hline 61 & 1 & 5 & 5 & $\mathbf{B}$ & 62 & 1 & 4 & 9 & B \\
\hline 63 & 1 & 6 & 7 & $B$ & 64 & 16 & 5 & 5 & B \\
\hline 65 & 1 & 1 & 10 & A & 66 & 1 & 3 & 2 & B \\
\hline 67 & 1 & 3 & 7 & $B$ & 68 & 1 & 15 & 42 & B \\
\hline 69 & 1 & 8 & 10 & B & 70 & 1 & 16 & 17 & B \\
\hline 71 & 1 & 10 & 20 & B & 72 & 1 & 3 & -1 & B \\
\hline 73 & 1 & 8 & 11 & B & 74 & 1 & 4 & 4 & B \\
\hline 75 & 1 & 4 & 15 & B & 76 & 1 & 3 & 5 & B \\
\hline 77 & 1 & 3 & 2 & B & 78 & 1 & 3 & 1 & B \\
\hline 79 & 1 & 1 & -1 & $\mathbf{A}$ & 80 & 6 & 3 & 12 & B \\
\hline 81 & 1 & 3 & 10 & B & 82 & 1 & 3 & 9 & B \\
\hline 83 & 1 & 2 & 4 & $\mathbf{A}$ & 84 & 1 & 3 & 2 & B \\
\hline 85 & 6 & 12 & 9 & B & 86 & 1 & 2 & 1 & A \\
\hline 87 & 1 & 2 & 1 & A & 88 & 1 & 2 & 1 & A \\
\hline 89 & 1 & 2 & 0 & A & & & & & \\
\hline
\end{tabular}

Table 1: Detailed data for scheduling example

\section{Stochastic Availability of Raw Material}

The LR scheduling algorithm of [9] assumes that raw material for the jobs to be scheduled is available when required. Since raw materials are usually procured from sources external to the machine shop, one can never be sure unless the raw material is in hand. The algorithm in [9] does handle the feature of delayed availability of raw material by specifying the earliest start times for individual jobs. But the earliest start times are specified in the very beginning when the algorithm is run. Consequently, the earliest start times cannot completely capture a stochastic environment where the raw material keeps arriving into the shop at time instants not known in advance. We propose the following method.

Let $S$ be the set of all jobs. Assume that $T_{0}, T_{1}, \cdots, T_{n}$ are the time instants during the time horizon $K$ at which raw material for jobs arrives into the machine shop. The instants $T_{0}, T_{1}, \cdots, T_{n}$, are not known in advance. Let $S_{0}, S_{1}, \cdots, S_{N}$ be the sets of jobs for which the raw material becomes available at $T_{0}, T_{1}, \cdots, T_{n}$, respectively. Assume further that $T_{0}=0$ so that $S_{0}$ is the set of jobs for which raw material is available at time zero. Table 2 shows a typical scenario for the 89-job example. The set $S_{0}$ in the table contains all the jobs not belonging to the sets $S_{1}$, $S_{2}, S_{3}$, and $S_{4}$.

\begin{tabular}{||c|c|c||}
\hline Time instant & Value of $T_{i}$ & $S_{i}$ \\
\hline$T_{0}$ & 0 & $S_{0}$ \\
$T_{1}$ & 10 & $\{5,9,21,30,34,41,63\}$ \\
$T_{2}$ & 25 & $\{62,76,84\}$ \\
$T_{3}$ & 28 & $\{3,43,65,81,82\}$ \\
$T_{4}$ & 100 & $\{13,25,68,71,75,80\}$ \\
\hline
\end{tabular}

Table 2. Data for delayed availability of raw material

Since the instants $T_{1}=10, T_{2}=25, T_{3}=28$ and $T_{4}=100$, are real-time instants which are not known in advance, they cannot be adequately specified by earliest start times. At time $T_{0}=0$, we first schedule, using the LR algorithm, all jobs in $S_{0}$, to obtain beginning times $\left\{B_{i}\right\}$ for all $i \in S_{0}$. The production commences and jobs in $S_{0}$ are scheduled according to $\left\{B_{i}\right\}$. At the instant $T_{1}$, raw material for jobs in $S_{1}$ becomes available, so there is a need to obtain an updated schedule that includes all jobs in $S_{1}$. At time $T_{1}$, the set $S_{0}$ can be partitioned into the following three sets.

$$
S_{01}=\left\{i \in S_{0}: B_{i}<T_{1} \text { and } B_{i}+t_{i} \leq T_{1}\right\}
$$




$$
\begin{gathered}
S_{02}=\left\{i \in S_{0}: B_{i}<T_{1} \text { and } B_{i}+t_{i}>T_{1}\right\} \\
S_{03}=\left\{i \in S_{0}: B_{i} \geq T_{1}\right\}
\end{gathered}
$$

Note in the above that $B_{i}$ and $t_{i}$ are the beginning time and processing time, respectively of job $i\left(i \in S_{0}\right)$. Jobs belonging to $S_{01}$ would already be processed by $T_{1}$ and pose no problem. Jobs belonging to $S_{02}$ would be undergoing processing at time $T_{1}$ whereas jobs in $S_{03}$ are not even initiated by time $T_{1}$. The rescheduling that we need to do will therefore include all jobs in $S_{03}$ and $S_{1}$ and possibly $S_{02}$. Whether or not we reschedule jobs in $S_{02}$ depends on whether we prefer to preempt the jobs in $S_{02}$ at time $T_{1}$ or we prefer to wait until the instant at which all jobs in $S_{02}$ are processed. In the former case, the jobs rescheduled belong to the set $S_{02} \cup S_{03} \bigcup S_{1}$, the rescheduling is done starting from $t=T_{1}$, and certain machine time and possibly materials are wasted. In the latter case, the jobs rescheduled belong to the set $S_{03} \cup S_{1}$, the rescheduling is done at $t=T_{1}+T_{02}$ where $T_{02}$ is the time needed for completing the jobs already initiated, and a higher weighted tardiness is to be expected. The rescheduling is again done using the $L R$ algorithm of [9]. The above procedure is repeated at the time instants $T_{2}, T_{3}, \cdots, T_{n}$.

\subsection{Numerical Example}

Consider the raw material arrival data given in Table 2. Recall that the 89 jobs specified in Table 1 have to be scheduled on to four machines. We first obtain the schedule, using the LR algorithm [9], for all jobs in the set $S_{0}$ (see Table 2). At time $t=10$, raw material for jobs in $S_{1}$ arrives and rescheduling becomes necessary. Assuming that we wait until all jobs that are already initiated at time $t=10$ to be completed, the rescheduling is done only at $t=18$.

\begin{tabular}{||l|r|r|r|r|r|r|r|r|r||}
\hline$M_{1}$ & 79 & 58 & 86 & 50 & 22 & 12 & 18 & 34 & 46 \\
& 54 & 9 & 40 & 64 & 79 & 73 & 3 & 65 & 80 \\
& 75 & & & & & & & & \\
$M_{2}$ & 55 & 1 & 7 & 21 & 41 & 15 & 59 & 29 & 4 \\
& 87 & 83 & 47 & 51 & 23 & 42 & 66 & 76 & 37 \\
& 74 & 33 & 43 & 13 & 68 & & & & \\
$M_{3}$ & 31 & 17 & 6 & 14 & 85 & 16 & 27 & 63 & 5 \\
& 19 & 10 & 48 & 11 & 52 & 88 & 2 & 77 & 44 \\
& 67 & 56 & 38 & 24 & 70 & 60 & 35 & 82 & 25 \\
$M_{4}$ & 36 & 20 & 30 & 32 & 45 & 49 & 53 & 89 & 72 \\
& 78 & 57 & 84 & 28 & 39 & 8 & 62 & 61 & 26 \\
& 81 & 71 & & & & & & & \\
\hline
\end{tabular}

Table 3: Final sequencing of jobs taking into account delayed availability of raw material

Table 3 shows the final schedule for all the jobs taking into account the raw material arrival sequence in Table
2. From Table 3 , we note the following. Jobs 30 and 63 which belong to $S_{1}$ are scheduled immediately due to their high weights. Jobs 62,76 , and 84 in $S_{2}$ are found to be delayed because of their late due dates. Since raw material for jobs in $S_{4}$ arrive only at $t=100$, the four machines will have to be idle starting from $t=85$ onwards, until $t=100$.

\section{Scrapping and Reworking of Jobs}

Quality control considerations entail inspection of processed jobs at frequent intervals to verify if the quality is acceptable. A typical inspection operation can have three outcomes: accept, reject, or rework. In case it is decided that a finished part is to be rejected or reworked, rescheduling becomes necessary. We propose the following scheme to handle such situations.

Assume that inspection operations are carried out at time instants $T_{1}, T_{2}, \cdots, T_{n}$. At time $T_{1}$, all parts processed until that time are inspected and parts to be rejected and parts to be reworked along with reworking times are decided. Rescheduling is done using the LR algorithm so as to include the parts identified for rejection and reworking. The due dates and weights of these parts can be assumed to be the original ones. If $S$ is the set of all jobs; $S_{1}$, the set of jobs processed by time $T_{1}$; and $S_{r}$ the set of jobs identified for rejection or reworking at $T_{1}$, then the set of jobs rescheduled at $T_{1}$ will be $\left(S-S_{1}\right) \cup S_{r}$. The procedure is repeated at all inspection epochs and we thus have an on-line procedure to handle scrapping and reworking of jobs.

\subsection{Numerical Example}

Table 4 shows a particular inspection program for the 89-job system of Table 1 . There are three inspection epochs, $T_{1}, T_{2}$, and $T_{3}$. At the epoch $T_{1}=20$, all jobs processed until then are inspected and it is found that job 16 is to be rejected and job 20 is to be reworked. Thus the reworking time for job 16 is 2 (original processing time $=2$ ). The reworking time for job 20 is decided to be 7 (original processing time $=10$ ). The rescheduling done at this epoch will therefore include these two jobs. Similarly, rescheduling is done as per the data in Table 4. Table 5 shows the overall schedule for all the 89 jobs, taking into account the scrapping and reworking. We find that job 20 is reworked immediately whereas job 16 is postponed by a significant amount. 


\begin{tabular}{||c|c|c||}
\hline Epoch & $i$ & Time \\
\hline 20 & 16 & 2 \\
20 & 20 & 7 \\
50 & 9 & 3 \\
50 & 49 & 4 \\
50 & 17 & 1 \\
50 & 23 & 3 \\
150 & 10 & 6 \\
150 & 35 & 5 \\
150 & 60 & 8 \\
150 & 26 & 9 \\
\hline
\end{tabular}

Table 4: Data for scrapping and reworking of jobs

\begin{tabular}{||l|r|r|r|r|r|r|r|r|r||}
\hline$M_{1}$ & 36 & 79 & $\mathbf{1 4}$ & 43 & 52 & 4 & 7 & 13 & 27 \\
& 29 & 87 & 22 & $\mathbf{4 2}$ & 67 & 7 & $\mathbf{1 7}$ & 38 & $\mathbf{9}$ \\
& $\mathbf{4 9}$ & 62 & 16 & 73 & 26 & $\mathbf{3 5}$ & & & \\
$M_{2}$ & 55 & 6 & 1 & 10 & 32 & 48 & 16 & 58 & 88 \\
& 83 & 51 & 12 & 18 & 84 & 76 & 44 & 81 & 37 \\
& 74 & 75 & 61 & 69 & 33 & 68 & $\mathbf{6 0}$ & & \\
$M_{3}$ & 31 & 30 & 41 & 19 & 15 & 45 & 11 & 49 & 53 \\
& 59 & 3 & $\mathbf{2 0}$ & 89 & 2 & 85 & 23 & 46 & 77 \\
& 56 & 82 & $\mathbf{2 3}$ & 8 & 40 & 64 & 70 & 35 & $\mathbf{1 0}$ \\
$M_{4}$ & 17 & 63 & 5 & 21 & 20 & 65 & 80 & 86 & 47 \\
& 50 & 34 & 72 & 78 & 54 & 57 & 66 & 28 & 39 \\
& 25 & 24 & 60 & $\mathbf{2 6}$ & & & & & \\
\hline
\end{tabular}

Table 5: Final sequencing taking into account reworking and scrapping of jobs. Bold faced jobs indicate scrapped or reworked parts.

\section{Effect of Set-ups in a Multi- class Production System}

In a multiclass production system, switchover times or set-up times can have a significant effect on the way parts are scheduled. Set-up costs would add a new dimension to the scheduling problem addressed by the LR algorithm [9] and incorporating set-up costs in the objective function will make the approach nondecomposable. Furthermore, the subproblems are not mutually independent any more, as jobs processed between successive set ups necessarily belong to the same class.

Assuming identical parallel machines to be available, we propose the following methodology for incorporating set up operations in the final schedules. First, the LR algorithm is used to schedule the given set of jobs in the available machines. This schedule will give a particular sequence of jobs on each machine. Let $J_{x_{1}}, J_{x_{2}}, \cdots, J_{x_{p}}$ be the sequence of jobs obtained for a specific machine. Let us assume these jobs belong to $C$ different classes and when the machine switches over from one job class to another, it incurs a set-up cost and also a set up time. The schedule $J_{x_{1}}, \cdots, J_{x_{p}}$, is nearly optimal if set-ups are not included. However if the same sequence is used and we include the set up costs, the resulting schedule can be very inefficient due to frequent changeovers. Our idea is to group the jobs in the sequence $J_{x_{1}}, \cdots, J_{x_{p}}$, in a suitable way, without disturbing the sequence, but at the same time minimize the total cost which will be the sum of the weighted tardiness and set-up cost.

We illustrate our approach by viewing the 89 -jobs of Table 1 as belonging to two different classes, say $A$ and $B$. The class information is shown in Table 1 . We have used the following criterion for classifying the jobs: jobs with processing time 1 or 2 are grouped as class A jobs and the rest of the jobs are designated as class $B$ jobs. We also assume that the switchover time from $A$ to $B$ is 6 units and that from $B$ to $A$ is 4 units.

Initially, the 89 jobs are scheduled using the LR algorithm onto the 4 machines. Let us look at the schedule for a particular machine. Let $J_{a_{1}}, J_{a_{2}}, \cdots, J_{a_{n_{a}}}$ be the class $A$ jobs in this schedule (in the same sequence as they appear in the schedule). Similarly, let $J_{b_{1}}, J_{b_{2}}, \cdots, J_{b_{n_{b}}}$ be the sequence of class B jobs in the schedule. First, we determine a range of suitable values for the number of switchovers using considerations such as:

1. Too many switchovers will make the set up costs dominate over the tardiness costs.

2. Too few switchovers will make the tardiness costs of delayed jobs substantial.

3. In a given set up, it is a good idea to process at least as many jobs having a total processing time equal to the set-up time.

Having chosen a certain number of switchovers, say $s$, we first look at the schedule having equal batch sizes for class A jobs and equal batch sizes for class B jobs. For example, in the above case, the batch size for class $A$ is chosen as $\frac{2 n_{a}}{s}$ and that for class $B$ as $\frac{2 n_{b}}{s}$. Calling $q_{a}$ and $q_{b}$ as the batch sizes for $\mathrm{A}$ and $\mathrm{B}$, the sequence will look like one of the following:

$$
\begin{aligned}
& J_{a_{1}}, \cdots, J_{a_{q_{a}}}, \text { switch, } J_{b_{1}}, \cdots, J_{b_{q_{b}}}, \text { switch, } \cdots \\
& J_{b_{1}}, \cdots, J_{b_{q_{b}}}, \text { switch, } J_{a_{1}}, \cdots, J_{a_{q_{a}}}, \text { switch, }, \cdots
\end{aligned}
$$

The objective function for such a sequence can be easily evaluated. The position of switch can now be changed by one or more positions to the left or right in a systematic way and in each case, the total cost of the resulting schedule can be evaluated. The procedure can be repeated until changes in the total cost 
keep decreasing and become negligible. This methodology can be repeated for other appropriate values of $s$ (number of switchovers). Schedules with set-ups can be obtained for other machines in an identical way.

\subsection{Numerical Example}

For the input data in Table 1, an LR schedule was obtained for each of the four machines. Assuming a switchover time of 6(4) units for a change from class A (class B) to class B (class A), a schedule with set-ups was obtained for each of the four machines. Table 6 shows the resulting schedules.

\begin{tabular}{||l|r|r|r|r|r|r|r|r|r||}
\hline$M_{1}$ & 36 & 43 & 6 & 13 & 21 & 16 & 58 & 86 & 83 \\
& 51 & 3 & 12 & 72 & 78 & 57 & 28 & 56 & 39 \\
& 74 & 24 & 71 & 60 & & & & & \\
$M_{2}$ & 30 & 79 & 5 & 14 & 41 & 10 & 32 & 11 & 48 \\
& 55 & 85 & 13 & 54 & 84 & 67 & 81 & 8 & 40 \\
& 69 & 26 & 35 & & & & & & \\
$M_{3}$ & 31 & 17 & 19 & 15 & 45 & 49 & 63 & 80 & 18 \\
& 42 & 66 & 76 & 9 & 38 & 29 & 87 & 47 & 22 \\
& 59 & 2 & 62 & 61 & 33 & 70 & & & \\
$M_{4}$ & 48 & 53 & 7 & 27 & 89 & 88 & 50 & 52 & 65 \\
& 34 & 20 & 23 & 46 & 77 & 44 & 82 & 37 & 75 \\
& 64 & 73 & 25 & 68 & & & & & \\
\hline
\end{tabular}

Table 6: Final sequencing taking into account set-ups

\section{Summary and Future Work}

Three extensions have been proposed in this paper to the Lagrangian relaxation based scheduling algorithm of Luh, Hoitomt, Max, and Pattipati [9], to address real-world features such as stochastic availability of raw material, scrapping and reworking of parts, and existence of significant set-up costs. The extensions proposed can be implemented easily. Numerical experiments on a 89 -job system have shown that these real-world features can often alter the original schedules in a significant way.

The issue of addressing the set-up problem needs to be examined in more detail. What we have provided is an efficient scheme for scheduling a two class production system with set-up times. The method needs to be extended to a multiclass system. A hybrid methodology that uses simulated annealing and Lagrangian relaxation has recently been developed to handle the set-up problem in systems with three or more job classes [11]. Other important questions in this context that need to be explored are: How to choose classes? How to choose batch sizes? Also, future work is needed to explore these extensions to multi-operation models and job shops.

\section{References}

1. M.L. Fisher, Lagrangian Relaxation Method for Solving Integer Programming Problems. Management Science, Volume 27, 1981, pp. 1-18.

2. S. French, Sequenching and Scheduling. Wiley, New York, 1982.

3. S.B. Gershwin, Hierarchical Flow Control: A Framework for Scheduling and Planning Discrete Events in Manufacturing Systems. Proceedings of the IEEE, Volume 77, Number 1, January 1989, pp. 195-209.

4. D.J. Hoitomt, P.B. Luh, and K.R. Pattipati, Job Shop Scheduling. Proceedings of First International Conference on Automation Technology, Taipei, Taiwan, July 1990, pp. 565-574.

5. D.J. Hoitomt, P.B. Luh, E. Max, and K.R. Pattipati, Scheduling Jobs with Simple Precedence Constraints on Parallel Machines. Chapter 20, in: Y.C. Ho (Editor), Discrete Event Systems, IEEE Press, 1991.

6. D.J. Hoitomt, P.B. Luh, and K.R. Pattipati, A Practical Approach to Job Shop Scheduling Problems, To appear in: IEEE Transactions on Robotics and Automation, 1993.

7. E.L. Lawler, J.K. Lenstra, A.H.G. Rinnooy Kan, and D.B. Shmoys, Sequencing and Scheduling: Algorithms and Complexity. Report No. BS- R8909, Centre for Mathematics and Computer Science, Amsterdam, November 1989.

8. D.G. Luenberger, Linear and Non-linear Programming. Second Edition: Addison-Wesley, Reading, Massachusetts, 1984.

9. P. Luh, D. Hoitomt, E. Max, and K. Pattipati, Schedule Generation and Reconfiguration for Parallel Machines. IEEE Transactions on Robotics and Automation, Volume 6, Number 6, December 1990, pp. 687696.

10. B.T. Polyak, Minimization of Unsmooth Functionals. USSR Computational Mathematics and Mathematical Physics, Volume 9, 1969, pp. 14-29.

11. R. Srigopal, Scheduling Multiclass Production Facilities using Lagrangian Relaxation. Master's Thesis, Department of Computer Science and Automation, Indian Institute of Science, Bangalore, January 1994.

12. N. Viswanadham and Y. Narahari, Performance Modeling of Automated Manufacturing Systems. Prentice Hall, Englewood Cliffs, New Jersey, 1992. 